\title{
Studying the Interrelationship amongst Various Lean Metrics to Construction Industry Through ISM Methodology
}

\author{
Arnav Jain \\ Techture Structures Private \\ Limited \\ Indore, India
}

\author{
Remica Aggarwal \\ School of Business, University of \\ Petroleum \& Energy Studies, \\ Dehradun, India
}

\author{
Veena Aggarwal \\ Recventures Education Services \\ Private Limited \\ Delhi, India
}

\begin{abstract}
Construction industry is the second largest sector in India. Due to its wide socio economic status, it has been a major area of research as well. Construction industry is known for heavy wastes during production, construction planning, administration etc. Lean is often characterized by minimization of waste. Present paper aims to explore the various lean metrics which helps to reduce wastages in construction thereby improving work quality in the product development process. It further studies the interrelationship amongst them using ISM methodology.
\end{abstract}

\section{Keywords}

ISM methodology; construction industry; lean metrics; wastages in construction

\section{INTRODUCTION}

Construction sector bags the second position in India only after agricultural sector. It primarily contributes towards the socio-economic development of a country and directly or indirectly impact many other industries and hence a house to many large scale, medium to small scale industrial, construction and manufacturing projects . Fast track projects with long and complex multiple supplier supply chains and extensive changes in process designs often results in complex flow management which if not checked timely, failed miserably. This failure does not come alone. It is accompanied by delays in goods delivery as well as cost and time overruns. Other factors responsible for such delays include lack of knowledge amongst workers, negligence towards work, choice of wrong construction process and improper material procurement, handling, supply and manufacturing. This results in backorders and sometimes lost sales as well i.e. a very high level of wastes and non value added activities add on to the production costs. This significant increase in waste as contended by lean construction institute [1] which usually contributes to around $57 \%$ of productive time waste can be handled by taking appropriate measures. This calls for appropriate practical ideas and techniques to be used in construction to help the project teams deal with wastages in construction with the optimum use of resources and this can be achieved by using lean construction and techniques.

\subsection{Lean construction and identification of causes of waste}

Lean is often characterized by minimization of waste. Various wastes in the construction industry have been categorize into headings such as mal-administration wastes (which includes lack of transparency, lack of team work , lack of experienced staff, lack of training programs, lack of risk management plans, poor safety etc.) ; design management wastes (inadequate time given for planning and design stage, design changes by clients, improper documentation of design data, lack of past project review and delay in drawing approval ; construction planning and site management waste (unnecessary work, rework, poor planning, lack of proper supervision, poor communication , waiting time, excessive inventory, human error etc.). Various lean tools which are frequently used in construction industry are value stream mapping for permitting systematic view of value flow process [2], integrated project delivery (IPD) which encourages communication and collaboration among participants [3], last planner system (LPS) which adds value through structuring the planning and ensuring proper flow process ([4]) etc.

\subsection{IT based applications for lean construction}

Since lean practices started with its applications and use in the manufacturing industry and gradually it got implemented to the distinguishing characteristics of the construction business [5]. Some of the oldest practices at the origin of lean construction are the last planner system, pull scheduling and just-in-time delivery [4]. Eventually it got extended to include the practices such as concurrent engineering and virtual design and construction (also known as building information modeling) and set based design [6]. Its application in construction industry can be compared to a train model in which the workers moves over the construction site completing the required activities. The key idea is that it is not possible to move only one coach independently, but all of them should move together. Some of the common lean practices are Building Information Modeling (BIM)/Virtual Design and Construction (VDC) (usually used to improve the efficiency of the design phase, reduce the time taken and quantity take off, increase the percentage planned-complete by a considerable amount, increases reliability and visibility through 3D/4D virtual models [7] ; cloud computing (usually used for rapid visualization of demand and supply data and for instant tracking of construction material status in the supply chain, for merging and synchronizing multiple sources of information for better communication among various stakeholders [8] ; Common Data Environment (CDE) ( used to collect, manage, share information and disseminate all relevant documents among various stakeholders [9] ; Data Mining ( helps to extract repeated and useful patterns from a large data set to predict the outcome of future events([10]; Geographical Information System (GIS) ( used for providing access to asset data within reasonable time for site feasibility analysis, and efficient use of equipment [11]; GPS Support 
System - GPS helps in tracking the whereabouts of fleet of assets in order to reduce lead-time at work sites with improved delivery and customer satisfaction ([12]); On-site vision tracking and use of CCTV (for effective positioning of personnel in construction sites, activity sequence analysis, enhancing pull flow mechanism, better localization of tools and material, detection of conflicts, visualization of waste and safety on site [13]; Virtual Reality (VR) and Augmented Reality (AR) and Web-Based Information System (for reducing processing time, increasing RFI transparency, enhancing flow reliability [14]. In addition to the various practices mentioned above, lean implementation also gets influenced by organizational culture factors $([15],[16])$. The establishment of a performance evaluation system is among the critical success factors for implementing Lean in manufacturing industries [17].

The objectives of the paper are to explore the various lean metrics widely used by construction industry in developing countries and to further study the inter-relationship amongst them through ISM methodology. Paper is organized as follows. Section 2 deals with literature review. Section 3 describes the ISM methodology. Section 4 discusses the case problem and constructs the ISM model and the diagraph. Section 5 discusses the managerial implications and directions for future research.

\section{LITERATURE REVIEW}

\subsection{Literature review on KPIs and metrics in construction industry}

Formerly, construction companies used financial measures to measure and evaluate their performance but over a period of time these measures became less important as management is more interested now days in up to date and mostly nonfinancial information to make better management decisions [18] and financial indicators are mostly lagging indicators. After a long dependence on financial measures, many studies and researches have been conducted to develop performance measurement frameworks that included financial and nonfinancial indicators. Often these indicators known as KPIs or metrics reflect the organization's perspectives as well as application specific perspectives such as project and supplier for construction [19]. Key performance indicators (KPIs) or metrics are compilations of data measures used to assess the performance of a construction operation [20]. KPIs play a key role in providing information on the performance of construction tasks, projects, and companies. Identification of key performance indicators often emerged as the applications of benchmarking. [21] mentioned productivity, safety , profitability , customer satisfaction, growth and predictability. [22] explored the key performance indicators or measuring construction success. As per construction industry research and information association (CIRIA) ${ }^{1}$, understanding client needs, design process, integration of design with SC internal cost/ time management, client / user satisfaction , innovation, risk, reuse of design experience and internal cost / time management are the important KPIs . [23] indicated the various criteria such as leadership , customer focus , stakeholder focus partnership and supplier management risk management, resource management internal stakeholder performance, external stakeholder performance organizational performance etc. from view point of contractors for success in construction industry . Similarly, [24] presents a computerized model for measuring and benchmarking the partnering performance of construction projects and proposes various KPIs such as time performance cost performance, quality performance, trust and respect, effective communication and innovation and improvement for measuring performance in construction industry. [25] illustrates the application of KPIs with respect to south eastern European construction. [26] presents various factors contributing to the success of equipment intensive sub contractors in construction. [27], [28],[29] critically evaluates the success factors for construction industry and building projects with respect to Malaysian building sector, Lithuania construction industry and Norwegian construction industry respectively. Kristensen et al. [30] presents the performance measurement indicators with respect to building design process. They found that punctuality, active participation, PPC , Proofing, time consumption , request for information , completeness and conformity, client satisfaction, collaboration, total cost and environment management are key performance indicators for achieving success in construction industry . [31] diagnose the impact of contractors' attributes on construction project success post construction.

\subsection{Literature on Lean construction metrics}

Lean metrics are quite popular in manufacturing academia ([32],[33], [34]). This study is focused on the effects of Lean as a consequence of its implementation rather than assessing the level of leanness of companies. Establishing a performance evaluation system can be part of the strategy for the success implementation of Lean practices [17]. The challenges in the Lean construction implementation do not come only from the adaptation of Lean to a project based setting, but implementing Lean has some challenges itself. [15] established a new set of principles for engineer-to-order production systems, which can be directly related to the construction industry and [17] named six critical success factors for the Lean implementation of small and medium companies as most of the subcontractors in the construction industry. The common factors found in the implementation process of Lean and performance measurement systems are the commitment and support from the management and the involvement of employees, either when creating indicators or developing Lean practices through their participation ([17],[35]-[37]). As seen in the interviews and confirming [38], project evaluation focused on costs as main factor, while other metrics such as schedule accomplishment, quality or other HSE factors were less valued. As per [39], evaluation framework proposed four areas of metrics in lean construction viz. core project metrics, customer metrics, workers metrics and environmental metrics. This paper focuses on the lean metrics proposed by [39] which are described as follows:

- Project metrics: Core project metrics contains the most common aspects currently used in the evaluation of projects such as schedule, cost, quality or HSE aspects. Major core project metrics are project completion time (PCT); project budget $(\mathrm{PB})$; average Per cent Planned Completed (PPC) of planned tasks (PPC) and warranty costs (WC) (This could be per meter cube).

- Customer metrics: These metrics can be the tabulated values obtained from results of a survey (usually on a scale of 1 to 10). This area is expected to be filled in the early phases of the project and it should contain the specific expectations of the final user, often represented by the project owner. This metrics includes predictable completion time $(\mathrm{PrCT})$; predictable results (PR) and low energy 
consumption (LEC) in use.

- Worker's Metrics: These metrics could be the tabulated values according to the results from evaluators' assessment of his workers. These metrics include safety (SFT); predictability (PRED); internal collaboration (IC) and external collaboration (EC); tidiness (TIDY) and absence rate (AR).

- Environment metrics: Environmental metrics are aimed to provide information about specific circumstances that could affect the development of the project. It is clear that extreme weather affect the working conditions, especially in construction. Establishing some thresholds value for temperature, rain or snow can facilitate the inclusion of its impact on the project. Obviously, employees still work behind those thresholds, but it is also clear that they would need breaks more often or require more time for the same tasks. Major metrics could be weather this includes temperature (TEMP) and rainfall (RFM) measurement and changes in terms of man hours (MH) (this includes flexibility and rework).

Therefore, 14 lean metrics viz. PCT , PB PPC, PrCT , PR, LEC , SFT , IC , EC , TIDY, AR , TEMP , RFM and MH have been recognized and their interrelationships have been studied using the ISM Methodology which has been explained in the next section.

\section{INTERPRETIVE STRUCTURAL MODELLING METHODOLOGY}

Interpretive Structural Modeling or ISM first proposed by Warfield [40] is a computer assisted learning process that enables the researcher to develop a map of the complex relationships between the many elements involved in a complex situation. In this technique a set of unique interrelated variables are structured in the form of a hierarchy graph called the diagraph. Its steps are as follows: Firstly, identify the relevant elements and establish a contextual relationship amongst them. Thereafter, develop a structural self-interaction matrix (SSIM) using V, A, X \& O where the symbols have the following meanings i.e. $\mathrm{V}$ for the relation from $\mathrm{i}$ to $\mathrm{j}$ but not in both directions; $A$ for the relation from $\mathrm{j}$ to $\mathrm{i}$ but not in both directions; $\mathrm{X}$ for both direction relations from $\mathrm{i}$ to $\mathrm{j}$ and $\mathrm{j}$ to $\mathrm{i}$; and $\mathrm{O}$ if the relation between the variables does not appear valid. Using SSIM, initial reachability matrix can be formed which has all values in binary form. A final reachability matrix is formed after removing transitivity from initial reachability matrix. From the final reachability matrix, the reachability set and antecedent set for each criterion and for each element is found [40]. The element for which the reachability and intersection sets are the same is the top-level element. At every iteration a top level element is identified which is removed in the next iteration. After all the elements have been identified at different level of hierarchy, a Mic-Mac analysis (based on the driving power and dependence power) and a diagraph can be formed.

\section{DEVELOPMENT OF ISM MODEL}

This section develops the ISM model for studying the interrelationships amongst the metrics in building and construction industry. The 14 lean metrics considered are project completion time (PCT); project budget $(\mathrm{PB})$; average PPC (PPC) and predictable completion time (PrCT); predictable results (PR) and low energy consumption (LEC) in use ; safety (SFT); internal collaboration (IC) and external collaboration (EC); tidiness (TIDY) and absence rate (AR) ; temperature (TEMP) and rainfall (RFM) measurement) and changes in terms of man hours (MH) (this includes flexibility and rework).

\subsection{Construction of Structural Self - Interaction Matrix (SSIM)}

This matrix gives the pair-wise relationship between two variables i.e. $i$ and $j$ based on VAXO. SSIM has been presented below in Fig 1.

\subsection{Construction of Initial Reachability \\ Matrix and final reachability matrix}

The SSIM has been converted in to a binary matrix called the initial reachability matrix shown in fig. 2 by substituting V, A, $\mathrm{X}, \mathrm{O}$ by 1 or 0 as per the case. After incorporating the transitivity, the final reachability matrix is shown below in the Fig 3.

Fig 1: SSIM matrix for pair wise relationship amongst barriers

\begin{tabular}{|c|c|c|c|c|c|c|c|c|c|c|c|c|c|c|c|}
\hline $\begin{array}{c}\text { Met } \\
\text { rics }\end{array}$ & & 1 & 2 & 3 & 4 & 5 & 6 & 7 & 8 & 9 & 10 & 11 & 12 & 13 & 14 \\
\hline & & $\begin{array}{c}\text { PC } \\
\text { T }\end{array}$ & $\begin{array}{c}\text { P } \\
\text { B }\end{array}$ & $\begin{array}{c}\text { PP } \\
\text { C }\end{array}$ & $\begin{array}{c}\text { PrC } \\
\text { T }\end{array}$ & PR & $\begin{array}{c}\text { LE } \\
\text { C }\end{array}$ & $\begin{array}{c}\text { SF } \\
\text { T }\end{array}$ & IC & EC & TID & AR & TE & RF & M \\
MP & M & H \\
\hline 1 & PCT & & A & A & A & A & O & A & A & A & O & A & A & A & A \\
\hline 2 & PB & & & A & A & A & A & A & X & A & A & A & A & A & A \\
\hline 3 & PPC & & & & V & V & A & A & A & A & O & A & A & A & A \\
\hline 4 & PrCT & & & & & A & O & A & A & A & O & A & A & A & A \\
\hline 5 & PR & & & & & A & A & A & A & A & A & A & A & A \\
\hline 6 & LEC & & & & & & & O & A & A & O & O & A & A & A \\
\hline 7 & SFT & & & & & & & & O & O & A & O & O & O & A \\
\hline 8 & IC & & & & & & & & & O & O & V & O & O & V \\
\hline 9 & EC & & & & & & & & & O & V & O & O & V \\
\hline
\end{tabular}




\begin{tabular}{|c|c|c|c|c|c|c|c|c|c|c|c|c|c|c|c|}
\hline 10 & TIDY & & & & & & & & & & & O & A & A & O \\
\hline 11 & AR & & & & & & & & & & & & A & A & V \\
\hline 12 & TEMP & & & & & & & & & & & & & V & V \\
\hline 13 & RFM & & & & & & & & & & & & & & V \\
\hline 14 & MH & & & & & & & & & & & & & \\
\hline
\end{tabular}

Fig 2: Initial reachability matrix

\begin{tabular}{|c|c|c|c|c|c|c|c|c|c|c|c|c|c|c|c|}
\hline Metric & & 1 & 2 & 3 & 4 & 5 & 6 & 7 & 8 & 9 & 10 & 11 & 12 & 13 & 14 \\
\hline & & PCT & $\begin{array}{l}\mathrm{P} \\
\mathrm{B}\end{array}$ & PPC & $\begin{array}{c}\mathrm{PrC} \\
\mathrm{T}\end{array}$ & PR & $\begin{array}{c}\mathrm{LE} \\
\mathrm{C}\end{array}$ & SFT & $\mathrm{IC}$ & $\mathrm{EC}$ & $\begin{array}{c}\text { TID } \\
Y\end{array}$ & AR & $\begin{array}{l}\mathrm{TE} \\
\mathrm{MP}\end{array}$ & $\begin{array}{l}\text { RF } \\
\mathrm{M}\end{array}$ & $\mathrm{MH}$ \\
\hline 1 & PCT & 1 & 0 & 0 & 0 & 0 & 0 & 0 & 0 & 0 & 0 & 0 & 0 & 0 & 0 \\
\hline 2 & $\mathrm{~PB}$ & 1 & 1 & 0 & 0 & 0 & 0 & 0 & 1 & 0 & 0 & 0 & 0 & 0 & 0 \\
\hline 3 & PPC & 1 & 1 & 1 & 1 & 1 & 0 & 0 & 0 & 0 & 0 & 0 & 0 & 0 & 0 \\
\hline 4 & PrCT & 1 & 1 & 0 & 1 & 0 & 0 & 0 & 0 & 0 & 0 & 0 & 0 & 0 & 0 \\
\hline 5 & PR & 1 & 1 & 0 & 1 & 1 & 0 & 0 & 0 & 0 & 0 & 0 & 0 & 0 & 0 \\
\hline 6 & LEC & 1 & 0 & 0 & 0 & 1 & 1 & 0 & 0 & 0 & 0 & 0 & 0 & 0 & 0 \\
\hline 7 & SFT & 1 & 1 & 1 & 1 & 1 & 0 & 1 & 0 & 0 & 0 & 0 & 0 & 0 & 0 \\
\hline 8 & $\mathrm{IC}$ & 1 & 1 & 1 & 1 & 1 & 1 & 0 & 1 & 0 & 0 & 1 & 0 & 0 & 1 \\
\hline 9 & $\mathrm{EC}$ & 1 & 1 & 1 & 1 & 1 & 1 & 0 & 0 & 1 & 0 & 1 & 0 & 0 & 1 \\
\hline 10 & TIDY & 1 & 0 & 0 & 0 & 1 & 0 & 1 & 0 & 0 & 1 & 0 & 0 & 0 & 0 \\
\hline 11 & $\mathrm{AR}$ & 1 & 1 & 1 & 1 & 1 & 0 & 0 & 0 & 0 & 0 & 1 & 0 & 0 & 1 \\
\hline 12 & TEMP & 1 & 1 & 1 & 1 & 1 & 1 & 0 & 0 & 0 & 1 & 1 & 1 & 1 & 1 \\
\hline 13 & RFM & 1 & 1 & 1 & 1 & 1 & 1 & 0 & 0 & 0 & 1 & 1 & 0 & 1 & 1 \\
\hline 14 & $\mathrm{MH}$ & 1 & 1 & 1 & 1 & 1 & 1 & 1 & 0 & 0 & 0 & 0 & 0 & 0 & 1 \\
\hline
\end{tabular}

Fig 3 : Final reachability matrix

\begin{tabular}{|c|c|c|c|c|c|c|c|c|c|c|c|c|c|c|c|c|}
\hline Metrics & & 1 & 2 & 3 & 4 & 5 & 6 & 7 & 8 & 9 & 10 & 11 & 12 & 13 & 14 & D.P \\
\hline & & PCT & $\mathrm{PB}$ & PPC & $\begin{array}{c}\mathrm{PrC} \\
\mathrm{T}\end{array}$ & PR & $\begin{array}{c}\mathrm{LE} \\
\mathrm{C}\end{array}$ & SFT & $\mathrm{IC}$ & $\mathrm{EC}$ & $\begin{array}{c}\text { TID } \\
Y\end{array}$ & AR & $\begin{array}{l}\mathrm{TE} \\
\mathrm{MP}\end{array}$ & $\begin{array}{c}\mathrm{RF} \\
\mathrm{M}\end{array}$ & $\mathrm{MH}$ & \\
\hline 1 & PCT & 1 & 0 & 0 & 0 & 0 & 0 & 0 & 0 & 0 & 0 & 0 & 0 & 0 & 0 & 1 \\
\hline 2 & PB & 1 & 1 & 0 & 0 & 0 & 0 & 0 & 1 & 0 & 0 & 0 & 0 & 0 & 0 & 3 \\
\hline 3 & PPC & 1 & 1 & 1 & 1 & 1 & 0 & 0 & 0 & 0 & 0 & 0 & 0 & 0 & 0 & 5 \\
\hline 4 & PrCT & 1 & 1 & 0 & 1 & 0 & 0 & 0 & 0 & 0 & 0 & 0 & 0 & 0 & 0 & 3 \\
\hline 5 & PR & 1 & 1 & 0 & 1 & 1 & 0 & 0 & 0 & 0 & 0 & 0 & 0 & 0 & 0 & 4 \\
\hline 6 & LEC & 1 & 0 & 1 & 0 & 1 & 1 & 0 & 0 & 0 & 0 & 0 & 0 & 0 & 0 & 4 \\
\hline 7 & SFT & 1 & 1 & 1 & 1 & 1 & 0 & 1 & 0 & 0 & 0 & 0 & 0 & 0 & 0 & 6 \\
\hline 9 & $\mathrm{EC}$ & 1 & 1 & 1 & 1 & 1 & 1 & 0 & 0 & 1 & 0 & 1 & 0 & 0 & 1 & 9 \\
\hline 10 & TIDY & 1 & 1 & 1 & 1 & 1 & 0 & 1 & 0 & 0 & 1 & 0 & 0 & 0 & 0 & 7 \\
\hline 11 & AR & 1 & 1 & 1 & 1 & 1 & 1 & 0 & 0 & 0 & 0 & 1 & 0 & 0 & 1 & 8 \\
\hline 12 & TEMP & 1 & 1 & 1 & 1 & 1 & 1 & 1 & 0 & 0 & 1 & 1 & 1 & 1 & 1 & 12 \\
\hline
\end{tabular}




\begin{tabular}{|c|c|c|c|c|c|c|c|c|c|c|c|c|c|c|c|c|}
\hline 13 & RFM & 1 & 1 & 1 & 1 & 1 & 1 & 1 & 0 & 0 & 1 & 1 & 0 & 1 & 1 & 11 \\
\hline 14 & MH & 1 & 1 & 1 & 1 & 1 & 1 & 1 & 0 & 0 & 0 & 0 & 0 & 0 & 1 & 8 \\
\hline & De.P & 14 & 12 & 10 & 11 & 11 & 7 & 5 & 2 & 1 & 3 & 5 & 1 & 2 & 6 & \\
\hline
\end{tabular}

D.P : Driving power ; De.P : dependence power

\subsection{Level Partition}

Table 3 : Iteration I

\begin{tabular}{|c|c|c|c|c|}
\hline $\begin{array}{l}\text { S. } \\
\text { No } \\
\text {. }\end{array}$ & $\begin{array}{l}\text { Reachabilit } \\
y_{\text {set }}\end{array}$ & $\begin{array}{l}\text { Antecedent } \\
\text { set }\end{array}$ & $\begin{array}{l}\text { Intersectio } \\
\text { n set }\end{array}$ & $\begin{array}{l}\text { Iteratio } \\
\text { n/ } \\
\text { Levels }\end{array}$ \\
\hline $\begin{array}{r}1 \\
.\end{array}$ & 1 & $\begin{array}{l}1,2,3,4,5,6,7,8, \\
9,10,11,12,13, \\
14\end{array}$ & 1 & \multirow{15}{*}{ I } \\
\hline 2. & 1,2 & $\begin{array}{l}2,3,4,5,7,8,9,1 \\
0,11,12,13,14\end{array}$ & 2 & \\
\hline 3. & $1,2,4,5$ & $\begin{array}{l}3,5,7,8,9,10,11 \\
, 12,13,14\end{array}$ & 5 & \\
\hline 4. & $1,2,3,4,5$ & $\begin{array}{l}3,7,8,9,10,11,1 \\
2,13,14\end{array}$ & 3 & \\
\hline 5. & $1,2,4$ & $\begin{array}{l}3,4,5,7,8,9,10, \\
11,12,13,14\end{array}$ & 4 & \\
\hline 6. & $1,3,5,6$ & $\begin{array}{l}6,8,9,11,12,13, \\
14\end{array}$ & 6 & \\
\hline 7. & $1,3,5,6,14$ & $\begin{array}{l}8,9,11,12,13,1 \\
4\end{array}$ & 14 & \\
\hline 8. & $1,3,5,6,7,14$ & $12,13,14$ & 14 & \\
\hline 9. & $\begin{array}{l}1,2,3,4,5,6,7, \\
8,10,14\end{array}$ & 8 & 8 & \\
\hline 10. & $\begin{array}{l}1,2,3,4,5,6,7, \\
10,11,12,13, \\
14\end{array}$ & 12 & 12 & \\
\hline 11. & $1,3,5,6,11,14$ & $8,9,11,12,13$ & 11 & \\
\hline 12. & $\begin{array}{l}1,2,3,5,6,10, \\
11,13,14\end{array}$ & 12,13 & 13 & \\
\hline 13. & $1,2,3,4,5,7$ & $7,10,12,13,14$ & 7 & \\
\hline 14. & $\begin{array}{l}1,2,3,4,5,6,9, \\
11,14\end{array}$ & 9 & 9 & \\
\hline 15. & 7,10 & $10,12,13$ & 10 & \\
\hline
\end{tabular}

From the final reachability matrix, reachability and final antecedent set for each factor are found. The element for which the reachability and intersection sets are same are the top-level element in the ISM hierarchy. After the identification of top level element, it is separated out from the other elements and the process continues for next level of elements. Reachability set, antecedent set, intersection set along with different level for elements have been shown below in table $\mathrm{V}$ to table XI.

Table 4 : Iteration II

\begin{tabular}{|c|c|c|c|c|}
\hline S.No. & $\begin{array}{c}\text { Reachabili } \\
\text { ty set }\end{array}$ & Antecedent set & $\begin{array}{c}\text { Intersection } \\
\text { set }\end{array}$ & $\begin{array}{c}\text { Itera } \\
\text { tion }\end{array}$ \\
\hline 2. & $\mathbf{2}$ & $\begin{array}{c}2,3,4,5,7,8,9,10 \\
, 11,12,13,14\end{array}$ & $\mathbf{2}$ & \\
\hline 3. & $2,4,5$ & $\begin{array}{c}3,5,7,8,9,10,11, \\
12,13,14\end{array}$ & 5 & \\
\hline 4. & $2,3,4,5$ & $\begin{array}{c}3,7,8,9,10,11,1 \\
2,13,14\end{array}$ & 3 & \\
\hline 5. & 2,4 & $\begin{array}{c}3,4,5,7,8,9,10,1 \\
1,12,13,14\end{array}$ & \multicolumn{2}{|c|}{4} \\
\hline
\end{tabular}

\begin{tabular}{|c|c|c|c|c|}
\hline 6. & $3,5,6$ & $\begin{array}{c}6,8,9,11,12,13 \\
14\end{array}$ & 6 & \multirow{10}{*}{ II } \\
\hline 7. & $3,5,6,14$ & $8,9,11,12,13,14$ & 14 & \\
\hline 8. & $3,5,6,7,14$ & $12,13,14$ & 14 & \\
\hline 9. & $\begin{array}{c}2,3,4,5,6,7 \\
8,10,14\end{array}$ & 8 & 8 & \\
\hline 10. & $\begin{array}{c}2,3,4,5,6,7 \\
10,11,12,13 \\
, 14\end{array}$ & 12 & 12 & \\
\hline 11. & $3,5,6,11,14$ & $8,9,11,12,13$ & 11 & \\
\hline 12. & $\begin{array}{c}2,3,5,6,10,1 \\
1,13,14\end{array}$ & 12,13 & 13 & \\
\hline 13. & $2,3,4,5,7$ & $7,10,12,13,14$ & 7 & \\
\hline 14. & $\begin{array}{c}2,3,4,5,6,9, \\
11,14\end{array}$ & 9 & 9 & \\
\hline 15. & 7,10 & $10,12,13$ & 10 & \\
\hline
\end{tabular}

Table 5 : Iteration III

\begin{tabular}{|c|c|c|c|c|}
\hline $\begin{array}{l}\text { Sr. } \\
\text { No. }\end{array}$ & $\begin{array}{c}\text { Reachability } \\
\text { set }\end{array}$ & $\begin{array}{c}\text { Antecedent } \\
\text { set }\end{array}$ & $\begin{array}{c}\text { Intersection } \\
\text { set }\end{array}$ & $\begin{array}{l}\text { Itera } \\
\text { tion }\end{array}$ \\
\hline 3. & 4,5 & $\begin{array}{c}3,5,7,8,9,10, \\
11,12,13,14\end{array}$ & 5 & \multirow{13}{*}{ III } \\
\hline 4. & $3,4,5$ & $\begin{array}{c}3,7,8,9,10,11 \\
, 12,13,14\end{array}$ & 3 & \\
\hline 5. & 4 & $\begin{array}{c}3,4,5,7,8,9,1 \\
0,11,12,13,1 \\
4\end{array}$ & 4 & \\
\hline 6. & $3,5,6$ & $\begin{array}{c}6,8,9,11,12,1 \\
3,14\end{array}$ & 6 & \\
\hline 7. & $3,5,6,14$ & $\begin{array}{c}8,9,11,12,13, \\
14\end{array}$ & 14 & \\
\hline 8. & $3,5,6,7,14$ & $12,13,14$ & 14 & \\
\hline 9. & $\begin{array}{c}3,4,5,6,7,8,10 \\
14\end{array}$ & 8 & 8 & \\
\hline 10. & $\begin{array}{c}3,4,5,6,7,10,11 \\
, 12,13,14\end{array}$ & 12 & 12 & \\
\hline 11. & $3,5,6,11,14$ & $8,9,11,12,13$ & 11 & \\
\hline 12. & $\begin{array}{c}3,5,6,10,11,13 \\
14\end{array}$ & 12,13 & 13 & \\
\hline 13. & $3,4,5,7$ & $\begin{array}{c}7,10,12,13,1 \\
4\end{array}$ & 7 & \\
\hline 14. & $3,4,5,6,9,11,14$ & 9 & 9 & \\
\hline 15. & 7,10 & $10,12,13$ & 10 & \\
\hline
\end{tabular}


Table 6 : Iteration IV

\begin{tabular}{|c|c|c|c|c|}
\hline $\begin{array}{l}\text { S.N } \\
\text { o. }\end{array}$ & $\begin{array}{l}\text { Reachabilit } \\
\text { y set }\end{array}$ & Antecedent set & $\begin{array}{l}\text { Intersecti } \\
\text { on set }\end{array}$ & $\begin{array}{c}\text { Iterati } \\
\text { on/ } \\
\text { Levels }\end{array}$ \\
\hline 3. & 5 & $\begin{array}{c}3,5,7,8,9,10,11 \\
12,13,14\end{array}$ & 5 & \multirow{12}{*}{ IV } \\
\hline 4. & 3,5 & $\begin{array}{c}3,7,8,9,10,11,1 \\
2,13,14\end{array}$ & 3 & \\
\hline 6. & $3,5,6$ & $\begin{array}{c}6,8,9,11,12,13 \\
14\end{array}$ & 6 & \\
\hline 7. & $3,5,6,14$ & $8,9,11,12,13,14$ & 14 & \\
\hline 8. & $3,5,6,7,14$ & $12,13,14$ & 14 & \\
\hline 9. & $\begin{array}{c}3,5,6,7,8,10 \\
14\end{array}$ & 8 & 8 & \\
\hline 10. & $\begin{array}{c}3,5,6,7,10,1 \\
1,12,13,14\end{array}$ & 12 & 12 & \\
\hline 11. & $3,5,6,11,14$ & $8,9,11,12,13$ & 11 & \\
\hline 12. & $\begin{array}{c}3,5,6,10,11, \\
13,14\end{array}$ & 12,13 & 13 & \\
\hline 13. & $3,5,7$ & $7,10,12,13,14$ & 7 & \\
\hline 14. & $\begin{array}{c}3,5,6,9,11,1 \\
4\end{array}$ & 9 & 9 & \\
\hline 15. & 7,10 & $10,12,13$ & 10 & \\
\hline
\end{tabular}

Table 7 : Iteration $\mathrm{V}$

\begin{tabular}{|c|c|c|c|c|}
\hline $\begin{array}{l}\text { Sr. } \\
\text { No. }\end{array}$ & $\begin{array}{c}\text { Reachability } \\
\text { set }\end{array}$ & $\begin{array}{l}\text { Antecedent } \\
\text { set }\end{array}$ & $\begin{array}{c}\text { Intersection } \\
\text { set }\end{array}$ & $\begin{array}{l}\text { Itera } \\
\text { tion }\end{array}$ \\
\hline 4. & 3 & $\begin{array}{c}3,7,8,9,10,11 \\
, 12,13,14\end{array}$ & 3 & \multirow{11}{*}{$\mathbf{V}$} \\
\hline 6. & 3,6 & $\begin{array}{c}6,8,9,11,12,1 \\
3,14\end{array}$ & 6 & \\
\hline 7. & $3,6,14$ & $\begin{array}{c}8,9,11,12,13 \\
14\end{array}$ & 14 & \\
\hline 8. & $3,6,7,14$ & $12,13,14$ & 14 & \\
\hline 9. & $3,6,7,8,10,14$ & 8 & 8 & \\
\hline 10. & $\begin{array}{c}3,6,7,10,11,12 \\
13,14\end{array}$ & 12 & 12 & \\
\hline 11. & $3,6,11,14$ & $8,9,11,12,13$ & 11 & \\
\hline 12. & $\begin{array}{c}3,6,10,11,13,1 \\
4\end{array}$ & 12,13 & 13 & \\
\hline 13. & 3,7 & $\begin{array}{c}7,10,12,13,1 \\
4\end{array}$ & 7 & \\
\hline 14. & $3,6,9,11,14$ & 9 & 9 & \\
\hline 15. & 7,10 & $10,12,13$ & 10 & \\
\hline
\end{tabular}

Table 8 : Iteration VI

\begin{tabular}{|c|c|c|c|c|}
\hline $\begin{array}{l}\text { Sr. } \\
\text { No. }\end{array}$ & $\begin{array}{c}\text { Reachability } \\
\text { set }\end{array}$ & Antecedent set & $\begin{array}{c}\text { Intersection } \\
\text { set }\end{array}$ & $\begin{array}{l}\text { Itera } \\
\text { tion }\end{array}$ \\
\hline 6. & 6 & $\begin{array}{c}6,8,9,11,12,13 \\
14\end{array}$ & 6 & \multirow{10}{*}{ VI } \\
\hline 7. & 6,14 & $8,9,11,12,13,14$ & 14 & \\
\hline 8. & $6,7,14$ & $12,13,14$ & 14 & \\
\hline 9. & $6,7,8,10,14$ & 8 & 8 & \\
\hline 10. & $\begin{array}{c}6,7,10,11,12,1 \\
3,14\end{array}$ & 12 & 12 & \\
\hline 11. & $6,11,14$ & $8,9,11,12,13$ & 11 & \\
\hline 12. & $6,10,11,13,14$ & 12,13 & 13 & \\
\hline 13. & 7 & $7,10,12,13,14$ & 7 & \\
\hline 14. & $6,9,11,14$ & 9 & 9 & \\
\hline 15. & 7,10 & $10,12,13$ & 10 & \\
\hline
\end{tabular}

Table 9 : Iteration VII

\begin{tabular}{|c|c|c|c|c|}
\hline $\begin{array}{l}\text { Sr. } \\
\text { No. }\end{array}$ & $\begin{array}{c}\text { Reachability } \\
\text { set }\end{array}$ & $\begin{array}{l}\text { Antecedent } \\
\text { set }\end{array}$ & $\begin{array}{c}\text { Intersection } \\
\text { set }\end{array}$ & $\begin{array}{l}\text { Itera } \\
\text { tion }\end{array}$ \\
\hline 7. & 14 & $\begin{array}{c}8,9,11,12,13, \\
14\end{array}$ & 14 & \multirow{9}{*}{ VII } \\
\hline 8. & 7,14 & $12,13,14$ & 14 & \\
\hline 9. & $7,8,10,14$ & 8 & 8 & \\
\hline 10. & $\begin{array}{c}7,10,11,12,13 \\
14\end{array}$ & 12 & 12 & \\
\hline 11. & 11,14 & $8,9,11,12,13$ & 11 & \\
\hline 12. & $10,11,13,14$ & 12,13 & 13 & \\
\hline 13. & 7 & $\begin{array}{c}7,10,12,13,1 \\
4\end{array}$ & 7 & \\
\hline 14. & $9,11,14$ & 9 & 9 & \\
\hline 15. & 7,10 & $10,12,13$ & 10 & \\
\hline
\end{tabular}

Table 10 : Iteration VIII

\begin{tabular}{|c|c|c|c|c|}
\hline $\begin{array}{l}\text { Sr. } \\
\text { No. }\end{array}$ & $\begin{array}{c}\text { Reachability } \\
\text { set }\end{array}$ & $\begin{array}{l}\text { Antecedent } \\
\text { set }\end{array}$ & $\begin{array}{c}\text { Intersection } \\
\text { set }\end{array}$ & $\begin{array}{l}\text { Itera } \\
\text { tion }\end{array}$ \\
\hline 9. & $7,8,10$ & 8 & 8 & \multirow{7}{*}{ VIII } \\
\hline 10. & $7,10,11,12,13$ & 12 & 12 & \\
\hline 11. & 11 & $8,9,11,12,13$ & 11 & \\
\hline 12. & $10,11,13$ & 12,13 & 13 & \\
\hline 13. & 7 & $7,10,12,13$ & 7 & \\
\hline 14. & 9,11 & 9 & 9 & \\
\hline 15. & 7,10 & $10,12,13$ & 10 & \\
\hline
\end{tabular}


Table 11 : Iteration IX

\begin{tabular}{|c|c|c|c|c|}
\hline $\begin{array}{c}\text { Sr. } \\
\text { No. }\end{array}$ & $\begin{array}{c}\text { Reachability } \\
\text { set }\end{array}$ & $\begin{array}{c}\text { Antecedent } \\
\text { set }\end{array}$ & $\begin{array}{c}\text { Intersection } \\
\text { set }\end{array}$ & $\begin{array}{c}\text { Itera } \\
\text { tion }\end{array}$ \\
\hline 9. & $7,8,10$ & 8 & 8 & \\
\cline { 1 - 4 } & $7,10,12,13$ & 12 & 12 & \multirow{2}{*}{ IX } \\
\cline { 1 - 4 } 12. & 10,13 & 12,13 & 13 & \\
\hline 13. & 7 & $7,10,12,13$ & 7 & \\
\hline 14. & 9 & 9 & 9 & \\
\hline 15. & 7,10 & $10,12,13$ & 10 & \\
\hline
\end{tabular}

Table 12 : Iteration $\mathrm{X}$

\begin{tabular}{|c|c|c|c|c|}
\hline $\begin{array}{l}\text { Sr. } \\
\text { No. }\end{array}$ & $\begin{array}{c}\text { Reachability } \\
\text { set }\end{array}$ & $\begin{array}{c}\text { Antecedent } \\
\text { set }\end{array}$ & $\begin{array}{c}\text { Intersection } \\
\text { set }\end{array}$ & $\begin{array}{l}\text { Itera } \\
\text { tion }\end{array}$ \\
\hline 9. & 8,10 & 8 & 8 & \multirow{5}{*}{$\mathbf{X}$} \\
\hline 10. & $10,12,13$ & 12 & 12 & \\
\hline 12. & 10,13 & 12,13 & 13 & \\
\hline 14. & 9 & 9 & 9 & \\
\hline 15. & 10 & $10,12,13$ & 10 & \\
\hline
\end{tabular}

Table 12 : Iteration XI

\begin{tabular}{|c|c|c|c|c|}
\hline $\begin{array}{c}\text { Sr. } \\
\text { No. }\end{array}$ & $\begin{array}{c}\text { Reachability } \\
\text { set }\end{array}$ & $\begin{array}{c}\text { Antecedent } \\
\text { set }\end{array}$ & $\begin{array}{c}\text { Intersection } \\
\text { set }\end{array}$ & $\begin{array}{c}\text { Itera } \\
\text { tion }\end{array}$ \\
\hline 9. & 8 & 8 & 8 & \multirow{2}{*}{ XI } \\
\hline 10. & 12,13 & 12 & 12 & \\
\hline 12. & $\mathbf{1 3}$ & 12,13 & $\mathbf{1 3}$ & \\
\hline 14. & 9 & 9 & 9 & \\
\hline 9. & 8 & 8 & 8 & \\
\hline
\end{tabular}

Table 13 :Iteration XII

\begin{tabular}{|c|c|c|c|c|}
\hline S.No. & $\begin{array}{c}\text { Reachability } \\
\text { set }\end{array}$ & $\begin{array}{c}\text { Anteced } \\
\text { ent set }\end{array}$ & $\begin{array}{c}\text { Intersectio } \\
\text { n set }\end{array}$ & $\begin{array}{c}\text { Iteration/ } \\
\text { Levels }\end{array}$ \\
\hline 9. & $\mathbf{8}$ & 8 & $\mathbf{8}$ & \multirow{2}{*}{ XII } \\
\hline 10. & $\mathbf{1 2}$ & 12 & $\mathbf{1 2}$ & \\
\hline 14. & $\mathbf{9}$ & 9 & $\mathbf{9}$ & \\
\hline
\end{tabular}

\subsection{Classification of factors}

The critical success factors described earlier are classified in to four clusters viz. autonomous factor, dependent factors, linkage factors and independent factors (mentioned in Table XIII below). As it can be seen that TIDY, SFT and LEC are autonomous criteria. Criteria TEMP, RFM, EC , IC , AR and $\mathrm{MH}$ are drivers . Criteria such as PPC, PR, PB and PrCT are dependent criteria. Fig. 4 below shows the driving power and dominance diagram.

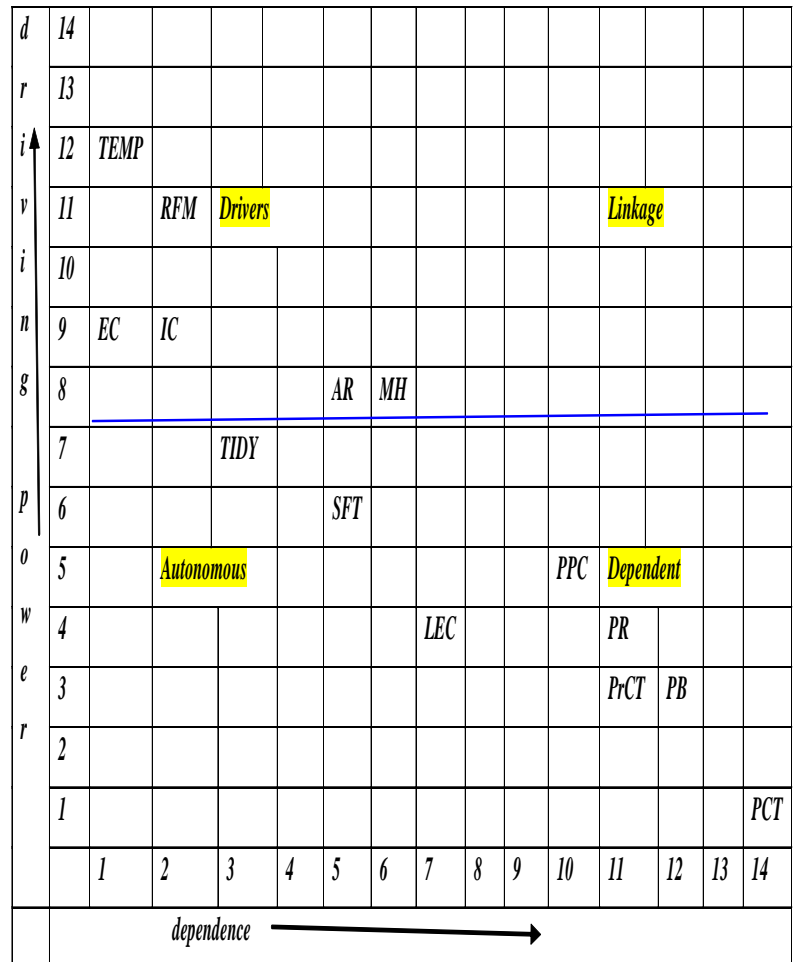

Fig . 4: Driving power and dependence diagram

\subsection{ISM model}

An ISM model is developed ( as shown in fig. 5 below ) after arranging the elements as per their interaction or dependence relationships.

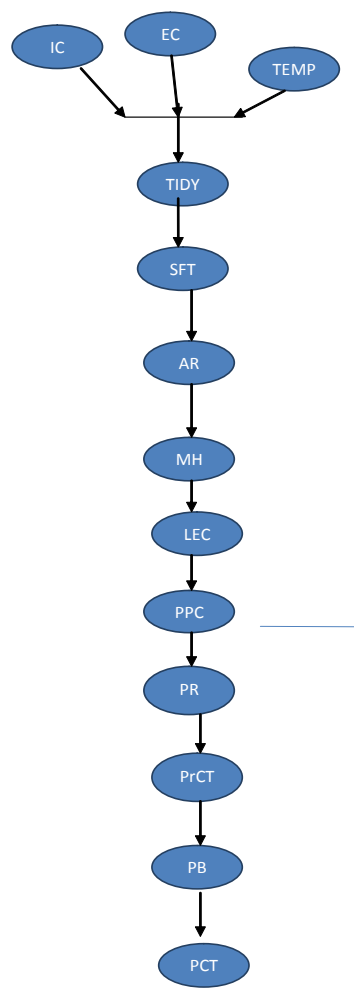

Fig 5: ISM diagraph 


\section{MANAGERIAL IMPLICATIONS \& CONCLUSIONS}

\subsection{Managerial implications}

This section focuses on managerial implications on using various lean practices in construction industry as well as assessing the construction lean metrics. Further this also provides directions for future research.

- Quite frequently, companies encourages the usage of lean practices or technique from strategic perspectives as well as practitioner's perspective provided the personnel have the required know how of its implementation.

- Further, implications of these techniques are related to other collateral issues such as improved cooperation, better defined responsibilities, less fights during the projects, greater commitment to the plan depends to a large extent on worker's commitment and motivation from the employer especially when the company requires working overtime and for longer periods of time [38].

- From the stakeholder's point of view as well, to invoke greater participation, needs for its successful implementation should be met [17].

- Using a specific framework for evaluating the metrics for project evaluation provides a base for assessing the impact of Lean practices in projects.

\subsection{Possible extensions and directions for future research}

- Other possible extension could be is of fuzzy logic based lean evaluation.

- Other perspectives from Lean implementation could add significant insight on the success factors. For example, focusing on organizational behavior could examine in more detail the motivation of people adopting Lean practices.

- Moreover, studying the role of performance measurement systems in dealing with uncertainty management can have a significant impact when measuring and implementing Lean, especially in case of considering the degree of projects' complexity [41]

- Lean construction framework with six sigma rating could also be a future direction [42]

\section{ACKNOWLEDGEMENT}

Authors are thankful to Prof. S.P Singh, Department of Management Studies for imparting knowledge on ISM methodology which helped authors substantially while writing this piece of work.

\section{REFERENCES}

[1] Ansah, R.H. , Sorooshian, S. \& Mustafa, S.B. 2016. Lean Construction: An Effective Approach Forproject Management, 11(3), ISSN 1819-6608 ARPN Journal of Engineering and Applied Sciences

[2] Pasqualini, F. \& Zawislak, P. (2005) Value Stream Mapping in Construction: A Case Study in a Brazilian Construction Company. The 13th International Group for Lean Construction Conference: Proceedings. Sydney,
Australia.

[3] Suttie, J. 2013. The impacts and effects of Integrated Project Delivery on participating organization with a focus on organization culture. The 21st International Group of Lean Construction. Fortaleza, Brazil.

[4] Ballard, G. \& Howell, G. 2003. An Update on Last Planner. The 11th Annual Conference of the International Group for Lean Construction. Virginia, USA.

[5] Koskela, L. 1997. Lean production in construction. Lean Construction, 1-9.

[6] Ballard, G. 2008. The lean project delivery system: An update. Lean Construction Journal, 1-19.

[7] Knotten, V. \& Svalestuen, F. 2014. Implementing Virtual Design and Construction (VDC) in Veidekke - Using Simple Metrics to Improve the Design Management Process. The 22nd Annual Conference of the International Group for Lean Construction. Oslo, Norway.

[8] Azambuja, M., Schnitzer, T., Sahin, M. \& Lee, F. 2013 Enabling Lean Supply with a Cloud Computing Platform - An Exploratory Case Study. The 21st Annual Conference of the International Group for Lean Construction. Fortaleza, Brazil.

[9] Specification for information management for the capital/delivery phase of construction projects using building information modelling. PAS 1192-2:2013.

[10] Danilevsky, M., Wang, C., Desai, N., Ren, X., Guo, J. \& Han, J. 2014. Automatic Construction and Ranking of Topical Key phrases on Collections of Short Documents.

[11] Maisuria, A. \& Rathod, H. (2013) GIS and its Application in Construction Industry. The International Journal of Advanced Research in Engineering, Science \& management.

[12] Simonsson, P. \& Carlsward, J. (2005) Evaluation of a GPS Support System for Fleet Management Control. The 13th Annual Conference of the International Group for Lean Construction. Sydney, Australia.

[13] Brilakis, I., Cordova, F. \& Clark, P. 2008. Automated 3D Vision Tracking for Project Control Support. The 15th EG-ICE International Workshop. Plymouth, UK.

[14] Chin, C. 2010 RFI Responsiveness of Paper-Based vs. Web-Based Information Processing Systems. The 18th Annual Conference of the International Group for Lean Construction. Haifa, Israel.

[15] Powell, D., Strandhagen, J. O., Tommelein, I., Ballard, G., \& Rossi, M. 2014. A New Set of Principles for Pursuing the Lean Ideal in Engineer-to-order Manufacturers. Procedia CIRP, 17(0), 571-576. doi: http://dx.doi.org/10.1016/j.procir.2014.01.137

[16] Cheung, S. O., Wong, P. S. P., \& Wu, A. W. Y. 2011. Towards an organizational culture framework in construction. International Journal of Project Management, 29(1), 33-44. doi: http://dx.doi.org/10.1016/j.ijproman.2010.01.014

[17] Bakås, O., Govaert, T., \& Van Landeghem, H. (2011). Challenges and success factors for implementation of lean manufacturing in european SMES. Paper presented 
at the 13th International conference on the Modern Information Technology in the Innovation Processes of the Industrial Enterprise (MITIP 2011).

[18] Bassioni, H. A., Price, A. D. F., \& Hassan, T. M. (2005) Building a conceptual framework for measuring business performance in construction: an empirical evaluation. Construction Management and Economics, 23(5), 495507. doi: 10.1080/0144619042000301401.

[19] Kagioglou, M., Cooper, R. and Aouad, G. 2001. Performance management in construction: a conceptual framework. Construction Management and Economics, 19(1), 85-95.

[20] Cox, R. F., Issa, R. R., \& Ahrens, D. 2003. Management's perception of key performance indicators for construction. Journal of construction engineering and management, 129(2), 142-151.

[21] Horta, I., Camanho, A.S., Costa, J.M., 2010 Performance assessment of construction companies integrating key performance indicators and data envelopment analysis. J. Constr. Eng. Manage. 136 (5), 581-594.

[22] Chan, A. P., \& Chan, A. P. 2004. Key performance indicators for measuring construction success. Benchmarking: an international journal, 11(2), 203-221.

[23] Bassioni, H., Hassan, T., \& Price, A. (2008). Evaluation and analysis of criteria and sub-criteria of a construction excellence model. Engineering, Construction and Architectural Management, 15(1), 21-41.

[24] Yeung, J. F. Y., Chan, A. P. C. and Chan, D. W. M. 2009. A computerized model for measuring and benchmarking the partnering performance of construction projects. Automation in Construction, 18(8), 1099-1113. doi: 10.1016/j.autcon.2009.07.003

[25] Ng, S. T., Tang, Z., \& Palaneeswaran, E. 2009. Factors contributing to the success of equipment-intensive subcontractors in construction. International Journal of Project Management, 27(7), 736-744. doi: 10.1016/j.ijproman.2008.09.006

[26] Radujkovic, M., Vukomanovic, M., \& Dunovic, I. B. 2010. Application of key performance indicators in South-Eastern European construction. Journal of Civil Engineering andManagement, 16(4), 521-530. doi: $10.3846 /$ jcem. 2010.58

[27] Al-Tmeemy, S. M. H. M., Abdul-Rahman, H., \& Harun, Z. 2011. Future criteria for success of building projects in Malaysia. International Journal of Project Management, 29(3), 337-348. doi: 10.1016/j.ijproman.2010.03.003

[28] Gudiene, N., Banaitis, A., \& Banaitiene, N. 2013. Evaluation of critical success factors for construction projects - an empirical study in Lithuania. International Journal of Strategic Property Management, 17(1), 21-31. doi: 10.3846/1648715x.2013.787128

[29] Hoseini, E. 2015. Project planning in the Norwegian construction industry. (Master in Project Management),
Norges Teknisk-Naturvitenskapelige Universitet, NTNU, Trondheim.

[30] Kristensen, K. H., Andersen, B. and Torp, O. 2013. Performance measurement in the building design process: a tool for building design managers. Journal of Design Research, 11(2), 148-167.

[31] Alzahrani, J. I., \& Emsley, M. W. 2013. The impact of contractors' attributes on construction project success: A post construction evaluation. International Journal of Project Management, 31(2), 313-322. doi: 10.1016/j.ijproman.2012.06.006

[32] Vimal, K., \& Vinodh, S. 2012. Leanness evaluation using IF-THEN rules. The International Journal of Advanced Manufacturing Technology, 63(1-4), 407-413.

[33] Vinodh, S., \& Balaji, S. 2011. Fuzzy logic based leanness assessment and its decision support system. International Journal of Production Research, 49(13), 4027-4041.

[34] Wan, H.-d., \& Frank Chen, F. 2008. A leanness measure of manufacturing systems for quantifying impacts of lean initiatives. International Journal of Production Research, 46(23), 6567-6584.

[35] Ballard, G., \& Howell, G. 1997. Implementing lean construction: improving downstream performance. Lean Construction, 111-125.

[36] Beatham, S., Chimay, A., Tony, T., \& Ian, H. (2005). An integrated business improvement system (IBIS) for construction. Measuring Business Excellence, 9(2), 4255. doi: 10.1108/13683040510602876

[37] Robinson, H. S., Anumba, C. J., Carrillo, P. M., \& AlGhassani, A. M. (2005). Business performance measurement practices in construction engineering organisations. Measuring Business Excellence, 9(1), 1322

[38] Andersen, B., Belay, A. M., \& Seim, E. A. 2012. Lean Construction Practices and its Effects: A Case Study at St Olav's Integrated Hospital, Norway. Lean Construction Journal, 122-149.

[39] Limon, D.H. 2015. Measuring Lean Construction: A Performance Measurement model supporting the implementation of Lean practices in the Norwegian construction industry, Project management thesis, Department of Production and Quality Engineering , Norwegian University of Science and Technology.

[40] Warfield, J.N. 1974. Developing interconnection matrices in structural modeling. IEEE Transactions on Systems, Man, and Cybernetics, (1), 81-87.

[41] Khanzode, A., Fischer, M., Reed, D., \& Ballard, G. 2006. A guide to applying the principles of virtual design $\&$ construction (VDC) to the lean project delivery process. CIFE, Stanford University, Palo Alto, CA.

[42] Raid, A. (2012). A lean construction framework with Six Sigma rating. International Journal of Lean Six Sigma, 3(4), 299-314. doi: 10.1108/20401461211284761 